\title{
On Multicolour Ramsey Numbers and Subset-Colouring of Hypergraphs
}

\author{
Bruno Jartoux ${ }^{1}$, Chaya Keller ${ }^{2}$, Shakhar Smorodinsky ${ }^{3}$, and Yelena Yuditsky ${ }^{4}$ \\ ${ }^{1}$ Department of Computer Science, Ben-Gurion University of the Negev, Be'er-Sheva, \\ Israel \\ jartoux@post.bgu.ac.il \\ ${ }^{2}$ Department of Computer Science, Ariel University, Ariel, Israel \\ chayak@ariel.ac.il \\ ${ }^{3}$ Department of Mathematics, Ben-Gurion University of the Negev, Be'er-Sheva, Israel \\ shakhar@math.bgu.ac.il \\ ${ }^{4}$ Département de Mathématique, Université libre de Bruxelles, Brussels, Belgium \\ yuditskyL@gmail.com
}

March 24, 2021

Bruno Jartoux: Research supported by the European Research Council (ERC) under the European Union's Horizon 2020 research and innovation programme (Grant agreement No. 678765) and by Grant 1065/20 from the Israel Science Foundation.

Chaya Keller: Research supported by Grant 1065/20 from the Israel Science Foundation. Shakhar Smorodinsky: Research supported by Grant 1065/20 from the Israel Science Foundation.

Yelena Yuditsky: Research supported by Belgian National Fund for Scientific Research (FNRS), through PDR grant BD-OCP.

\begin{abstract}
For $n \geq s>r \geq 1$ and $k \geq 2$, write $n \rightarrow(s)_{k}^{r}$ if every hyperedge colouring with $k$ colours of the complete $r$-uniform hypergraph on $n$ vertices has a monochromatic subset of size $s$. Improving upon previous results by Axenovich et al. [1] and Erdôs et al. 9 , we show that

$$
\text { if } r \geq 3 \text { and } n \nrightarrow(s)_{k}^{r} \text { then } 2^{n} \nrightarrow(s+1)_{k+3}^{r+1} .
$$

This yields an improvement for some of the known lower bounds on multicolour hypergraph Ramsey numbers.

Given a hypergraph $H=(V, E)$, we consider the Ramsey-like problem of colouring all $r$-subsets of $V$ such that no hyperedge of size $\geq r+1$ is monochromatic. We provide upper and lower bounds on the number of colours necessary in terms of the chromatic number $\chi(H)$. In particular we show that this number is $O\left(\log ^{(r-1)}(r \chi(H))+r\right)$.
\end{abstract}

\section{Introduction}

Even though Ramsey theory has attracted much attention from its inception almost a century ago, many questions remain elusive. This paper is primarily concerned with lower bounds for (multicolour, hypergraph) Ramsey numbers and the methods that yield them. 
Notations. For any natural number $n \in \mathbf{N}$, put $\llbracket n \rrbracket=\{0, \ldots, n-1\}$. For any set $S$ and $k \in \mathbf{N}$, the set $[S]^{k}$ is $\{T \subset S:|T|=k\}$, i.e., the set of all $k$-subsets of $S$. We write $\mathcal{P}(S)$ for the powerset of $S$. If $f: A \rightarrow B$ is a function and $X \subset A$, we write $f$ " $X=\{f(x): x \in X\}$ instead of the more usual (outside set theory) but ambiguous $f(X)$. Throughout the paper, $\log$ is the binary logarithm (although the choice of base is inconsequential in most places).

Definition 1.1 (Rado's arrow notation). Given $k \geq 2, n>r \geq 1$, an $r$-subset $k$-colouring of $\llbracket n \rrbracket$ is a function $f:[\llbracket n \rrbracket]^{r} \rightarrow \llbracket k \rrbracket$. A set $X \subset \llbracket n \rrbracket$ is monochromatic (under $f$, in the colour $i \in \llbracket k \rrbracket)$ if $[X]^{r} \subset f^{-1}(i)$, or equivalently $f "[X]^{r}=\{i\}$. For $k$ integers $\left(s_{i}\right)_{i \in \llbracket k \rrbracket}$ all satisfying $n \geq s_{i}>r$, we write

$$
n \rightarrow\left(s_{0}, \ldots, s_{k-1}\right)^{r},
$$

or more concisely $n \rightarrow\left(s_{i}\right)_{i \in \llbracket k \rrbracket}^{r}$, to mean that for every $f:\left[\llbracket n \rrbracket \rrbracket^{r} \rightarrow \llbracket k \rrbracket\right.$ there is a colour $i \in \llbracket k \rrbracket$ in which a subset of $\llbracket n \rrbracket$ of size $s_{i}$ is monochromatic. When $s_{0}=s_{1}=\cdots=s_{k-1}=s$ (the "diagonal case") this is further abbreviated to $n \rightarrow(s)_{k}^{r}$.

The logical negation of any arrow relation is written similarly, replacing $\rightarrow$ with $\nrightarrow$.

Our main result is as follows.

Theorem 1.2. Fix integers $k \geq 2$ and $n>r \geq 3$ and $k$ integers $\left(s_{i}\right)_{i \in \llbracket k \rrbracket}$ all satisfying $n \geq s_{i} \geq$ $r+1$.

$$
\text { If } n \nrightarrow\left(s_{i}\right)_{i \in \llbracket k \rrbracket}^{r} \text { then } 2^{n} \nrightarrow(s_{0}+1, \ldots, s_{k-1}+1, \underbrace{r+2, \ldots, r+2}_{\begin{array}{c}
\eta(r) \text { terms, } \\
\text { each } r+2
\end{array}})^{r+1},
$$

with the integer

$$
\eta(r)= \begin{cases}1 & \text { if } r=3 \\ 2 & \text { if } r>3 \text { is even } \\ 3 & \text { if } r>3 \text { is odd }\end{cases}
$$

This result extends to transfinite cardinals, strengthening results of Erdôs et al. 7, Chap. $24]$ in certain ranges of parameters (Appendix A).

Let $n \geq s>r \geq 1$ and $k \geq 2$. The (multicolour, hypergraph, diagonal) Ramsey number ${ }^{1}$ $r_{k}(s ; r)$ is the smallest $n$ for which $n \rightarrow(s)_{k}^{r}$. The fact that these numbers exist is Ramsey's 1930 theorem 14 .

As a corollary to Theorem 1.2, we obtain improved lower bounds for multicolour Ramsey numbers. The tower functions are defined by $\operatorname{twr}_{1}(x)=x$ and $\operatorname{twr}_{r+1}(x)=2^{\operatorname{twr}_{r}(x)}$.

Corollary 1.3. There are absolute constants $\alpha \simeq 1.678$ and $\beta$ such that for $r=3$ and any $k \geq 4$ or for $r \geq 4$ and $k \geq\lfloor 5 r / 2\rfloor-5$, we have:

$$
r_{k}(r+1 ; r)>\operatorname{twr}_{r}\left(\frac{\alpha}{2} \cdot\left(k-\frac{5 r}{2}\right)+\beta\right),
$$

and for $r=3$ and any $k \geq 2$ or for $r \geq 4$ and $k \geq\lfloor 5 r / 2\rfloor-7$,

$$
r_{k}(r+2 ; r)>\operatorname{twr}_{r}\left(\alpha \cdot\left(k-\frac{5 r}{2}\right)+\beta\right) .
$$

\footnotetext{
${ }^{1}$ Different authors use different notations: cf. $r_{k}\left(K_{s}^{r}\right)$ [1, $r_{r}(s ; k)[4], R_{k}(s ; r)$ [13].
} 
Subset colouring in hypergraphs. Our second result addresses a hypergraph colouring problem. Given a hypergraph $H$ and $r \in \mathbf{N}$, we are interested in the minimum number $k=k(H ; r)$ for which there exists a $k$-colouring of all $r$-subsets of vertices without any monochromatic hyperedge of size $\geq r+1$. Note that if all the hyperedges in $H$ are of size at least 2 , then $k(H ; 1)=\chi(H)$, the standard vertex chromatic number of $H$ (i.e. the least number of colours in a colouring of $V$ in which no hyperedge is monochromatic).

In a work on simplicial complexes, Sarkaria related this parameter (which he called the weak $r$-th chromatic number), to embeddability properties [15, 16, 17].

We show (Theorem 4.2) that for any $H$, the number of colours $k(H ; r)$ is not much larger than the corresponding number of colours $k\left(K_{r \chi(H)}^{(r+1)} ; r\right)$ where the hypergraph $K_{r \chi(H)}^{(r+1)}$ is the complete $(r+1)$-uniform on $r \chi(H)$ vertices. Note that $\chi\left(K_{r \chi(H)}^{(r+1)}\right)=\chi(H)$. This resembles the Erdôs-Stone theorem [8] (the chromatic number of a graph essentially controls its Turán number). Hence finding $k(n, r)=\max \{k(H ; r): \chi(H)=n\}$ for any $n, r$, is essentially equivalent to the problem of finding the Ramsey number $r_{k^{\prime}}(r+1 ; r)$ for an appropriate value of $k^{\prime}$.

With our lower bound on multicolour Ramsey numbers and the previously known upper bound, we obtain (Corollary 4.7):

$$
\Omega\left(\frac{\log ^{(r-1)}(r n)}{\log ^{(r)}(r n)}\right)<k(n, r)<O\left(\log ^{(r-1)}(r n)+r\right) .
$$

\subsection{Previous results}

The values of $r_{k}(s ; r)$ remain unknown except for several simple cases; in fact, for $r \geq 3$ only $r_{2}(4 ; 3)=13$ is known 12 . For general $r$ the only known bounds are

$$
\operatorname{twr}_{r}\left(c^{\prime} k\right) \leq r_{k}(s ; r) \leq \operatorname{twr}_{r}(c k \log k)
$$

for sufficiently large $s$, with $c$ and $c^{\prime}$ functions of $s$ and $r[6,7]$. See the book by Graham, Rothschild, and Spencer [11] for background on finite Ramsey theory and the survey by Radziszowski [13 for recent bounds.

Some lower bounds on Ramsey numbers are obtained through stepping-up lemmata. The following (negative) stepping-up lemma attributed is due Erdôs and Hajnal [11]. It transforms lower bounds on $r_{k}(\cdot ; r)$ into lower bounds on $r_{k}(\cdot ; r+1)$, as follows:

$$
\text { if } r \geq 3 \text { and } n \nrightarrow(s)_{k}^{r} \text { then } 2^{n} \nrightarrow(2 s+r-4)_{k}^{r+1},
$$

and, for stepping up from 2 to 3 :

$$
\text { if } n \nrightarrow(s)_{k}^{2} \text { then } 2^{n} \nrightarrow(s+1)_{2 k}^{3},
$$

where the number of colours doubles. Conlon, Fox, and Sudakov 44 improved (5):

$$
\text { if } r \geq 4 \text { and } n \nrightarrow(s)_{k}^{r} \text { then } 2^{n} \nrightarrow(s+3)_{k}^{r+1} \text {. }
$$

Furthermore for odd $r$ or for $k \geq 3$, they obtain

$$
\text { if } r \geq 4 \text { and } n \nrightarrow(s)_{k}^{r} \text { then } 2^{n} \nrightarrow(s+2)_{k}^{r+1} \text {. }
$$


Repeated application of this last lemma alone will not guarantee the lower bound of (4) where $s$ and $r$ are both large and $s<2 r$. For example, it cannot deal with the case where the size of the hyperedges grows by exactly 1 . This is why Axenovich et al. [1] implicitly establish the following variant:

$$
\text { if } r \geq 2 \text { and } n \nrightarrow(s)_{k}^{r} \text { then } 2^{n} \nrightarrow(s+1)_{2 k+2 r-4}^{r+1},
$$

which implies, in the left inequality of (4), that for any $s>r$ and any $k>r 2^{r}$, we have $r_{k}(s ; r)>\operatorname{twr}_{r}\left(\frac{k}{2^{r}}\right)$.

We mention one more earlier result by Erdôs et al. [9, Lemma 24.1]:

$$
\text { if } r \geq 3 \text { and } n \nrightarrow(s)_{k}^{r} \text { then } 2^{n} \nrightarrow(s+1)_{k+2^{r}+2^{r-1}-4}^{r+1} \text {. }
$$

\subsection{Organization of the paper}

Section 2 establishes our stepping-up lemma (Theorem 1.2). In Section 3 we apply said lemma to improve the lower bounds for several multicolour hypergraph Ramsey numbers. Section 4 deals with subset-colouring of hypergraphs. Appendix A extends the stepping-up lemma to infinite cardinals. Appendix B explains our starting bound on $r_{k}(3 ; 2)$.

\section{$2 \quad$ A stepping-up lemma}

In this section we prove Theorem 1.2. To this end let $k, n, r,\left(s_{i}\right)_{i \in \llbracket k \rrbracket}$ be as in the hypotheses. In particular, there exists $f_{r}:[\llbracket n \rrbracket]^{r} \rightarrow \llbracket k \rrbracket$ under which, for every $i \in \llbracket k \rrbracket$, no set of size $s_{i}$ is monochromatic in colour $i$. We will use $f_{r}$ to construct $f_{r+1}:\left[\llbracket 2^{n} \rrbracket\right]^{r+1} \rightarrow \llbracket k+\eta(r) \rrbracket$ under which, for every $i \in \llbracket k \rrbracket$, no set of size $s_{i}+1$ is monochromatic in colour $i$, and no set of size $r+2$ is monochromatic in either of the additional colours $k, k+1, \ldots, k+\eta(r)-1$.

\subsection{Preliminary results and definitions}

Before proceeding to the proof of the theorem, we need several definitions and results.

Splitting indices. For every natural number $n$, let $d(n) \subset \mathbf{N}$ be the unique finite set of integers such that $n=\sum_{i \in d(n)} 2^{i}$. In other words, $d(n)$ is the set of non-zero indices in the binary representation of $n$. Given a finite set $S$ of natural numbers, $|S| \geq 2$, its first splitting index is

$$
s(S)=\max \{i \in \mathbf{N}: \exists x, y \in S: i \in d(x) \backslash d(y)\} .
$$

That is, $s(S)$ is the index of the most significant bit where two elements in $S$ differ in their binary representation.

The first splitting index partitions $S$ into two disjoint, non-empty subsets $S_{0}=\{x \in$ $S: s(S) \notin d(x)\}$ and $S_{1}=\{x \in S: s(S) \in d(x)\}$ with $\max S_{0}<\min S_{1}$. This partition is unique and exists as soon as $|S| \geq 2$; we use the notation $\left(S_{0} \mid S_{1}\right)$ to denote the set $S$ where the partition $S_{0} \cup S_{1}$ is the partition obtained at the first splitting index.

Observation 2.1. Let $S=\left(S_{0} \mid S_{1}\right)$ be a set of at least two natural numbers. If $\emptyset \neq L \subset S_{0}$ and $\emptyset \neq R \subset S_{1}$ then $s(L \cup R)=s(S)$ and $L \cup R=(L \mid R)$. 
Caterpillars. We define a family $\mathcal{C} \subset \mathcal{P}(\mathbf{N})$, whose elements we call caterpillars (due to their resemblance with the caterpillar trees of graph theory), as follows: The empty set and all singletons are caterpillars. A finite set of 2 or more natural integers, with partition $\left(S_{0} \mid S_{1}\right)$, is a caterpillar if and only if at least one of $S_{0}$ and $S_{1}$ is a singleton and the other is a caterpillar.

For example, the reader may check that $\{0,1,2,4\}=(((\{0\} \mid\{1\}) \mid\{2\}) \mid\{4\}) \in \mathcal{C}$ and $\{0,1,2,3\}=(\{0,1\} \mid\{2,3\}) \notin \mathcal{C}$, by considering binary representations of the integers. One can depict the sequence of splits introducing the partitions using a full binary tree (see Figure 1). Caterpillars are those subsets whose binary tree possesses a dominating root-to-leaf path, that is, the vertex set of the tree can be partitioned into a path and a collection of vertices where each vertex has a neighbour on the path.
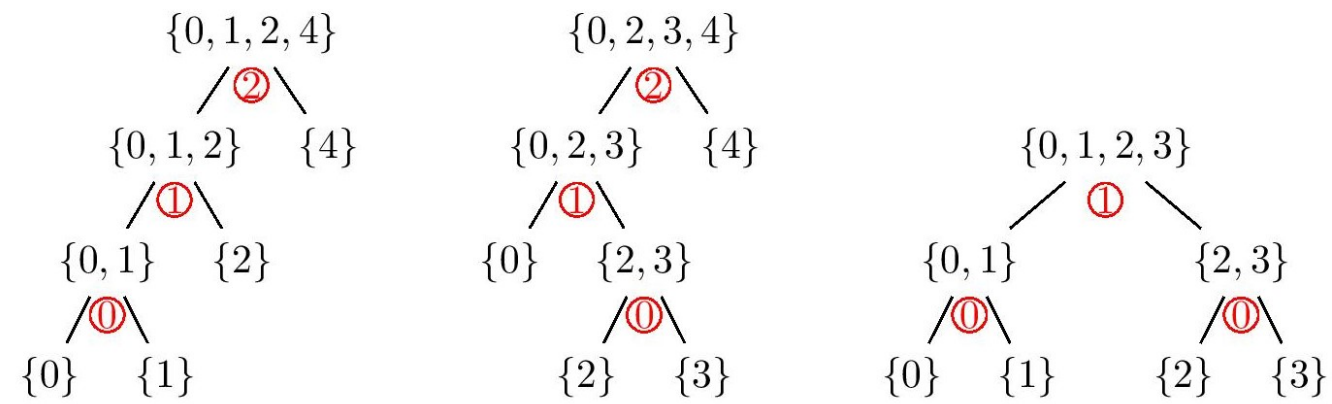

Figure 1: The tree structures of two caterpillars (left) and a non-caterpillar (right). The splitting indices are marked in red and circled.

The proofs by Erdôs et al. [9] or Axenovich et al. [1] use a smaller class of thin sets, namely, the caterpillars whose binary tree representations only have leaves for their proper left subtrees.

Observation 2.2. Let $S \subset \mathbf{N}$ be a finite set. The following are equivalent:

1. $S \notin \mathcal{C}$.

2. There is a subset $(L \mid R) \subset S$ with $|L|,|R| \geq 2$.

3. There is a subset $(L \mid R) \subset S$ with $|L|=|R|=2$.

As a simple consequence, $\mathcal{C}$ is transitive: if $S \in \mathcal{C}$ then $\mathcal{P}(S) \subset \mathcal{C}$.

Proof. The implication (1) $\Longrightarrow(2)$ can be easily proved by induction on $|S|$. Indeed, if $\left(S_{0} \mid S_{1}\right) \notin \mathcal{C}$ then either $S_{0} \notin \mathcal{C}$, or $S_{1} \notin \mathcal{C}$, or $\left|S_{0}\right|,\left|S_{1}\right| \geq 2$.

The implication $(2) \Longrightarrow(3)$ holds by Observation 2.1 .

To show (3) $\Longrightarrow(1)$, assume that (3) holds but (1) does not. By (3), there are four integers $a<b<c<d$ such that $(\{a, b\} \mid\{c, d\}) \subset S \in \mathcal{C}$. By the definition of $\mathcal{C}, S=\left(S_{0} \mid S_{1}\right)$ where either $\left|S_{0}\right|=1$ or $\left|S_{1}\right|=1$, without loss of generality, $\left|S_{0}\right|=1$. It must be the case that $S_{0} \cap\{a, b, c, d\}=\emptyset$ as otherwise $\left|S_{0}\right|>1$. Indeed, assume without loss of generality that $a \in S_{0}$, then $c \notin S_{0}$ but because $(\{a, b\} \mid\{c, d\}) \subset S$ we get that $b \in S_{0}$. Hence $\{a, b, c, d\} \subseteq S_{1}$ and by induction on the size of the set we consider we get a contradiction to (3).

With each caterpillar $S \in \mathcal{C}$ we associate a subset of indices $\delta(S)=\{s(\{x, y\}): x, y \in S, x \neq$ $y\}$. For example, the reader may check that $\delta(\{1,3,6,31\})=\{1,2,4\}$. 
Observation 2.3. If $S \in \mathcal{C} \backslash\{\emptyset\}$ then $|\delta(S)|=|S|-1$.

Proof. Simple induction using Observation 2.1.

Observation 2.4. For every $S \in \mathcal{C} \backslash\{\emptyset\}$ and $r \in \mathbf{N}$, we have $[\delta(S)]^{r}=\delta "[S]^{r+1}$.

Proof. Note that $[S]^{r+1} \subset \mathcal{C}$, so the right-hand term is well-defined.

For $r=0$ or $|S|=1$ this is clear: the equality becomes $\{\emptyset\}=\{\emptyset\}$ if $r=0$ or $\emptyset=\emptyset$ if $r>0$ and $|S|=1$. For larger $|S|$ and $r$, let $x \in S$ be such that $S=(L \mid\{x\}), L \in \mathcal{C} \backslash\{\emptyset\}$ and $s(L)<s(S)$. Then $\delta(S)=\{s(S)\} \cup \dot{\cup} \delta(L)$, where the union is disjoint. Thus

$$
[\delta(S)]^{r}=\left\{\{s(S)\} \cup D: D \in[\delta(L)]^{r-1}\right\} \cup \dot{\cup}[\delta(L)]^{r} .
$$

By induction on $r$ and the size of the set considered this is equal to

$$
[\delta(S)]^{r}=\left\{\{s(S)\} \cup \delta(Y): Y \in[L]^{r}\right\} \cup \dot{\cup}\left\{\delta(Y): Y \in[L]^{r+1}\right\}
$$

Thus,

$$
\begin{aligned}
{[\delta(S)]^{r} } & =\left\{\delta(Y \dot{\cup}\{x\}): Y \in[L]^{r}\right\} \dot{\cup}\left\{\delta(Y): Y \in[L]^{r+1}\right\} \\
& =\left\{\delta(Y): Y \in[S]^{r+1}\right\}=\delta "[S]^{r+1} .
\end{aligned}
$$

Each finite set $S=(L \mid R)$ of natural numbers that is not a caterpillar has a type $t(S) \in \mathbf{N} \times \mathbf{N}$, defined by induction as follows:

$$
t((L \mid R))= \begin{cases}(|L|,|R|) & \text { if }|L|,|R| \geq 2 \\ t(L) & \text { if }|R|=1 \\ t(R) & \text { if }|L|=1\end{cases}
$$

For example, $t(\{0,1,2,3,4,8\})=t(\{0,1,2,3\})=(2,2)$.

Observation 2.5. Let $S$ be a finite, nonempty set of integers.

(1) If $S$ has type $(p, q)$ with $p \geq 3$ then some subset of $S$ of cardinality $|S|-1$ has type $(p-1, q)$ (respectively $q \geq 3$ and $(p, q-1)$ ).

(2) If $S$ has type $(2, q)$ then some subset of $S$ of cardinality $|S|-1$ is a caterpillar or has type $\left(p^{\prime}, q^{\prime}\right)$ with $p^{\prime}+q^{\prime} \leq q$ (respectively $(p, 2)$ and $\left.p^{\prime}+q^{\prime} \leq p\right)$.

(3) If $S$ has type $(p, q)$ with $p+q<|S|$ then some subset of $S$ of cardinality $|S|-1$ also has type $(p, q)$.

(4) The caterpillars are exactly the finite subsets of $\mathbf{N}$ without subsets of type $(2,2)$.

Proof. (1) We prove by induction on $|S|$. If $|S|=p+q$ then $S=(L \mid R)$ with $|L|=p$ and $|R|=q$. Let $x$ be any element of $L$, then by Observation 2.1 the set $S \backslash\{x\}=(L \backslash\{x\}, R)$ has type $(p-1, q)$.

If $|S|>p+q$, then without loss of generality $S=(L \mid\{x\})$ with $t(L)=(p, q)$. By induction, there is $L^{\prime} \subset L$ with $\left|L^{\prime}\right|=|L|-1=|S|-2$ and $t\left(L^{\prime}\right)=(p-1, q)$. Then $L^{\prime} \cup\{x\} \subset S$ with $\left|L^{\prime} \cup\{x\}\right|=|S|-1$, and $t\left(L^{\prime} \cup\{x\}\right)=t\left(L^{\prime}\right)=(p-1, q)$. 
(2) By the same induction argument as above, it suffices to consider the case $|S|=2+q$. Then $S=(\{x, y\} \mid R)$ with $x \neq y$ and $|R|=q$. Hence by Observation 2.1, $S \backslash\{x\}=(\{y\} \mid R)$. If $R$ is a caterpillar, then $S \backslash\{x\}$ is a caterpillar, otherwise $S \backslash\{x\}$ has type $\left(p^{\prime}, q^{\prime}\right)$ with $p^{\prime}+q^{\prime} \leq|R|=q$.

(3) In this case, w.l.o.g., $S=(\{x\} \mid R)$ with $R$ of type $(p, q)$. Then $S \backslash\{x\}$ is of type $(p, q)$.

(4) Direct reformulation of Observation 2.2 (1),(3).

\subsection{Description and validity of the colouring.}

Description. We can now define our colouring $f_{r+1}:\left[\llbracket 2^{n} \rrbracket\right]^{r+1} \rightarrow \llbracket k+3 \rrbracket$. Let $S \in\left[\llbracket 2^{n} \rrbracket\right]^{r+1}$.

If $S \in \mathcal{C}$ then $\delta(S) \in[\llbracket n \rrbracket]^{r}$, and we let $f_{r+1}(S)=f_{r}(\delta(S)) \in \llbracket k \rrbracket$. Otherwise $S \notin \mathcal{C}$ has a type $t(S)=(p, q)$ with $p, q \geq 2$ and $p+q \leq r+1$, and we let

$$
f_{r+1}(S)= \begin{cases}k & \text { if } p+q=r+1, p \text { even } \\ k+1 & \text { if } p+q<r+1, p+q \text { even } \\ 0 & \text { if } p+q=r+1, p \text { odd and } r \text { odd } \\ k+2 & \text { if } p+q=r+1, p \text { odd and } r \text { even } \\ 1 & \text { if } p+q<r+1, p+q \text { odd }\end{cases}
$$

Validity. We detail the proof that this colouring has the expected properties for $r$ odd. The case of even $r$ is similar and easier. Let $X \subset \llbracket 2^{n} \rrbracket$ be monochromatic in colour $i$ under $f_{r+1}$. We will show that $|X| \leq s_{i}$ (if $i \in \llbracket k \rrbracket$ ) or $|X| \leq r+1$ (for $i \in\{k, k+1, k+2\}$ ). As $r+1 \leq s_{i}$ we may already assume $|X|>r+1$.

First consider the case $X \in \mathcal{C}$. Then by Observation 2.2 $[X]^{r+1} \subset \mathcal{C}$, and thus

$$
\{i\}=f_{r+1} "[X]^{r+1}=f_{r} "\left(\delta "[X]^{r+1}\right) \subset \llbracket k \rrbracket,
$$

so we have to show $|X| \leq s_{i}$. By Observation 2.4, the set $\delta(X) \subset \llbracket n \rrbracket$ is monochromatic in colour $i$ under $f_{r}$, as

$$
f_{r} "[\delta(X)]^{r}=f_{r} "\left(\delta "[X]^{r+1}\right)=\{i\} .
$$

This implies by the hypothesis on $f_{r}$ and Observation 2.3 that $|X|-1=|\delta(X)|<s_{i}$, as required.

Now consider the case $X \notin \mathcal{C}$. We can assume $|X|=r+2$. The set $X$ has a type $(P, Q)$ with $P, Q \geq 2$ and $P+Q \leq r+2$. We consider several cases covering all possible values of $P$ and $Q$, and we argue that in each case two $(r+1)$-subsets of $X$ receive distinct colours under $f_{r+1}$, i.e. $\left|f_{r+1} "[X]^{r+1}\right| \geq 2$.

- If $P+Q=r+2$ and $P, Q \geq 3$, then by Observation 2.5 (1), $X$ contains $(r+1)$ subsets $S, S^{\prime}$ of types $(P-1, Q)$ and $(P, Q-1)$, respectively, so also the pair of colours $\{0, k\}=\left\{f_{r+1}(S), f_{r+1}\left(S^{\prime}\right)\right\}$.

- If $(P, Q)=(2, r)$ : on one hand, by Observation 2.5 (1), there is an $(r+1)$-subset $S$ with the type $(2, r-1)$ and $f_{r+1}(S)=k$. On the other hand, by Observation 2.5 (2), there is an $(r+1)$-subset $S^{\prime}$ which is either a caterpillar, so $f_{r+1}\left(S^{\prime}\right) \in \llbracket k \rrbracket$, or of a type $(x, y)$ with $x+y \leq r$, so $f_{r+1}\left(S^{\prime}\right) \in\{1, k+1\}$.

- If $(P, Q)=(r, 2)$ : symmetric to the previous case, because $r-1$ is even. 
- If $P+Q \leq r+1$ and $P \geq 3$, then by Observation 2.5 (3), $X$ contains an $(r+1)$-subset $S$ of type $(P, Q)$ and by Observation $2.5(1)$ an $(r+1)$-subset $S^{\prime}$ of type $(P-1, Q)$. Since $S^{\prime}$ is not a caterpillar and $P-1+Q<r+1, f_{r+1}\left(S^{\prime}\right) \in\{1, k+1\}$. If $f_{r+1}(S) \in\{0, k\}$ then we are done. Otherwise, $f_{r+1}(S) \in\{1, k+1\}$, and the distinct parities of $P+Q$ and $P-1+Q$ give $\left\{f_{r+1}(S), f_{r+1}\left(S^{\prime}\right)\right\}=\{1, k+1\}$

- If $P+Q \leq r+1$ with $Q \geq 3$, same argument as the previous case.

- If $(P, Q)=(2,2)$ : because $r+2 \geq 5$, by Observation 2.5 (3), there is an $(r+1)$-subset $S$ of type $(2,2)$, whose colour is either $k$ (if $r=3$ ) or $k+1$. On the other hand, by Observation 2.5 (2), there is an $(r+1)$-subset $S^{\prime}$ which is a caterpillar, and so is coloured with some colour between 0 and $k-1$.

Observation 2.6. For odd $r$ the function $f_{r+1}$ does not use the colour $k+2$, and in the case of $r=3$ the function $f_{4}$ also does not use the colour $k+1$.

This completes the proof of Theorem 1.2 . We conclude with an observation which will be useful in Section 3 .

Observation 2.7. If $n \nrightarrow(3)_{k}^{2}$ then $2^{n} \nrightarrow(5)_{k}^{3}$.

Proof. First observe that every set of five natural numbers includes a caterpillar of size four. Let the elements of $S \in[\mathbf{N}]^{5}$ be $a, b, c, d, e$. If $S=(\{a\} \mid\{b, c, d, e\})$ then $\{a, b, c, d\} \in \mathcal{C}$. (Note that any 3 -subset of $\mathbf{N}$ is a caterpillar.) If $S=(\{a, b\} \mid\{c, d, e\})$ then $\{a, c, d, e\} \in \mathcal{C}$. Other cases are symmetric.

As in the proof of Theorem 1.2 , the colours in $\llbracket k \rrbracket$ suffice for colouring $\left[\llbracket 2^{n} \rrbracket\right]^{3}$ such that any caterpillar $S \subset \llbracket 2^{n} \rrbracket$ with $|S|=4$ is not monochromatic. Any 5 -subset of $\llbracket 2^{n} \rrbracket$ contains such an $S$, and thus is itself not monochromatic under the same colouring.

\section{Lower bounds on multicolour hypergraph Ramsey numbers}

Erdős, Hajnal and Rado obtained the first bounds on $r_{k}(s ; r)[6,7]$. Note the following:

Observation 3.1. The quantity $r_{k}(s ; r)$ is nondecreasing in $s$ and in $k$. The quantity $r_{k}(r+1 ; r)$ is nondecreasing in $r$.

Theorem 3.2 (Erdôs and Rado[7], Erdôs, Hajnal, and Rado[6]). Let $r \geq 2$. There exists $s_{0}(r)$ such that:

- For any $s>s_{0}$, we have $r_{k}(s ; r) \geq \operatorname{twr}_{r}\left(c^{\prime} k\right)$,

- For any $s>r$, we have $r_{k}(s ; r) \leq \operatorname{twr}_{r}(c k \log k)$,

where $c^{\prime}=c^{\prime}(s, r)$ and $c=c(s, r) \leq 3(s-r)$.

Unlike the upper bound, the lower bound holds only for sufficiently large values of $s$. Duffus, Leffman and Rödl [5] gave a tower-function lower bound for all $s \geq r+1$, but said bound, $\operatorname{twr}_{r-1}\left(c^{\prime \prime} k\right)$, is significantly weaker than the bound of Theorem 3.2 . Conlon, Fox, and Sudakov [4] proved that the lower bound of Theorem 3.2 holds whenever $s \geq 3 r$. Axenovich et al. [1] matched the lower bound of Theorem 3.2 for all $s>r$, but only for sufficiently large $k$. 
Theorem 3.3 (Axenovich et al.). For any $s>r \geq 2$ and any $k>r 2^{r}$, we have

$$
r_{k}(s ; r)>\operatorname{twr}_{r}\left(\frac{k}{2^{r}}\right) .
$$

In particular the results of [4] give $r_{k}(s ; 3) \geq 2^{2^{c^{\prime} k}}$ for some constant $c^{\prime}$ whenever $s \geq 9$, and the results of $|1|$ give $r_{k}(4 ; 3)>2^{2^{k / 8}}$ for all $k>24$.

We prove that the lower bound of Theorem 3.2 holds for values of $k$ much closer to $r$ than in Theorem 3.3, and also improve the constant inside the tower function.

Corollary (1.3). There are absolute constants $\alpha \simeq 1.678$ and $\beta$ such that for $r=3$ and any $k \geq 4$ or for $r \geq 4$ and $k \geq\lfloor 5 r / 2\rfloor-5$, we have:

$$
r_{k}(r+1 ; r)>\operatorname{twr}_{r}\left(\frac{\alpha}{2} \cdot\left(k-\frac{5 r}{2}\right)+\beta\right),
$$

and for $r=3$ and any $k \geq 2$ or for $r \geq 4$ and $k \geq\lfloor 5 r / 2\rfloor-7$,

$$
r_{k}(r+2 ; r)>\operatorname{twr}_{r}\left(\alpha \cdot\left(k-\frac{5 r}{2}\right)+\beta\right) .
$$

Proof. Fix some integers $K \geq 0$ and $n, s \geq 4$ such that $n \nrightarrow(s)_{K+2}^{3}$. Through either $2 t+1$ or $2 t+2$ repeated applications of Theorem 1.2 we get, for any $t \in \mathbf{N}$,

$$
\begin{aligned}
& \operatorname{twr}_{2 t+2} n \nrightarrow(s+2 t+1)_{K+5 t+3}^{4+2 t} \nrightarrow \\
& \text { and } \operatorname{twr}_{2 t+3} n \nrightarrow(s+2 t+2)_{K+5 t+5}^{5+2 t}
\end{aligned}
$$

Equivalently, in terms of Ramsey numbers,

$$
\begin{aligned}
r_{K+5 t+3}(s+2 t+1 ; 4+2 t) & >\operatorname{twr}_{2 t+2} n \\
\text { and } r_{K+5 t+5}(s+2 t+2 ; 5+2 t) & >\operatorname{twr}_{2 t+3} n .
\end{aligned}
$$

It is known (see Appendix $B$ that there are absolute constants $C_{0}>0$ and $C_{1}=1073^{1 / 6} \simeq$ 3.199 such that, for every integer $L \geq 0$,

$$
\left\lceil C_{0} \cdot C_{1}^{L+2}\right\rceil \nrightarrow(3)_{L+2}^{2} .
$$

From there we have both $2^{\left\lceil C_{0} \cdot C_{1}^{L+2}\right\rceil} \nrightarrow(4)_{2 L+4}^{3}$ and $2^{\left\lceil C_{0} \cdot C_{1}^{L+2}\right\rceil} \nrightarrow(5)_{L+2}^{3}$, which establishes the Theorem for $r=3$. The first relation follows from (6) and the second from Observation 2.7 . Insert these parameters in $(15)$ and $(16)$ to obtain respectively

$$
\begin{aligned}
& \operatorname{twr}_{2 t+4}\left(\log C_{0}+(L+2) \log C_{1}\right)<r_{2 L+5 t+5}(5+2 t ; 4+2 t), r_{L+5 t+3}(6+2 t ; 4+2 t), \\
& \operatorname{twr}_{2 t+5}\left(\log C_{0}+(L+2) \log C_{1}\right)<r_{2 L+5 t+7}(6+2 t ; 5+2 t), r_{L+5 t+5}(7+2 t ; 5+2 t) .
\end{aligned}
$$

Any even $r \geq 4$ is of the form $r=2 t+4$ (so $\lfloor 5 r / 2\rfloor=5 t+10)$ and thus (17) yields:

$$
\begin{aligned}
& \operatorname{twr}_{r}\left(\log C_{0}+(L+2) \log C_{1}\right)<r_{2 L+\lfloor 5 r / 2\rfloor-5}(r+1 ; r) \leq r_{(2 L+1)+\lfloor 5 r / 2\rfloor-5}(r+1 ; r), \\
& \operatorname{twr}_{r}\left(\log C_{0}+(L+2) \log C_{1}\right)<r_{L+\lfloor 5 r / 2\rfloor-7}(r+2 ; r),
\end{aligned}
$$

where the rightmost inequality in the first line follows from Observation 3.1. The same equations are also obtained for odd $r \geq 5$, this time by setting $r=2 t+5$ in (18). This proves (2) for all values $k \geq\lfloor 5 r / 2\rfloor-5$ (respectively (3) and $\lfloor 5 r / 2\rfloor-7$ ).

To the best of our knowledge, the current best lower bound for $r_{k}(5 ; 3)$ (for large $k$ ) is $\operatorname{twr}_{3}(k+O(1))\left[2\right.$. Compare with our $r_{k}(5 ; 3)>\operatorname{twr}_{3}(1.678 k+O(1))$. 


\section{Subset-colouring of hypergraphs}

Recall that, given a hypergraph $H=(V, E)$ and $r \in \mathbf{N}$, the integer $k=k(H ; r)$ is the least number of colours for which there is a colouring $[V]^{r} \rightarrow \llbracket k \rrbracket$ without any monochromatic hyperedge $e \in E$ of size $\geq r+1$, and $k(n, r)=\max \{k(H ; r): \chi(H)=n\}$. As noted by Sarkaria [17], the problem of determining $k(n, r)$ is closely related to the "classical" Ramsey problem discussed in previous sections. Indeed, for $n \in \mathbf{N}$, consider the complete $(r+1)$-uniform hypergraph on $r n$ vertices $K_{r n}^{(r+1)}$. Clearly, $\chi\left(K_{r n}^{(r+1)}\right)=n$, and we have

Observation 4.1. For any $n, r \in \mathbf{N}$,

$$
k\left(K_{r n}^{(r+1)} ; r\right)=\min \left\{k: r_{k}(r+1 ; r)>r n\right\} .
$$

Proof. By definition, $k\left(K_{r n}^{(r+1)} ; r\right)$ is the least $k$ for which there is a colouring $[\llbracket r n \rrbracket]^{r} \rightarrow \llbracket k \rrbracket$ without any monochromatic $(r+1)$-subset, i.e. such that $r n \nrightarrow(r+1)_{k}^{r}$, or equivalently such that $r n<r_{k}(r+1 ; r)$.

Our main result in this section is that the values $k(n, r)$ and $k\left(K_{r n}^{(r+1)} ; r\right)$ are very close to each other:

Theorem 4.2. For any positive integers $n$ and $r$,

$$
k\left(K_{r n}^{(r+1)} ; r\right) \leq k(n, r) \leq k\left(K_{r n}^{(r+1)} ; r\right)+5 .
$$

Theorem 4.2 will follow from Theorem 4.3 below. From the above Observation and Theorem we conclude that the problem of finding $k(n, r)$ is essentially equivalent to the problem of finding the Ramsey number $r_{k^{\prime}}(r+1 ; r)$ for an appropriate value of $k^{\prime}$.

Theorem 4.3. Let $r, k \geq 2$ and let $n$ be such that $n \nrightarrow(r+1)_{k}^{r}$. If the hypergraph $(V, E)$ admits a vertex-colouring $V \rightarrow \llbracket n \rrbracket$ under which no hyperedge of size $\geq r+1$ is monochromatic, then there is an r-subset colouring $[V]^{r} \rightarrow \llbracket k+f(r) \rrbracket$ such that no hyperedge of size $\geq r+1$ is monochromatic. Here

$$
f(r)= \begin{cases}1 & \text { if } r=2, \\ 3 & \text { if } r=3 \\ 4 & \text { if } r \geq 4 \text { and } r+1 \text { is prime } \\ 5 & \text { otherwise. }\end{cases}
$$

Remark 4.4. In Theorem 4.3 we require only hyperedges of size at least $(r+1)$ not to be monochromatic. A stronger but more natural condition is for $(V, E)$ to have vertex chromatic number at most $n$.

Proof of Theorem 4.2. Let $k=k\left(K_{r n}^{(r+1)} ; r\right)$, so that $r n \nrightarrow(r+1)_{k}^{r}$ and, by monotony, $n \nrightarrow$ $(r+1)_{k}^{r}$. Theorem 4.3 then states that any hypergraph with vertex chromatic number $n$ has an $r$-subset colouring with $k+5$ colours and no monochromatic hyperedge of size $r+1$, i.e. $k(n, r) \leq k+5$. The left inequality of 20 is by definition.

Remark 4.5. The two-sided relation with Ramsey numbers in Theorem 4.2 holds only for $k(n, r)$. For a specific hypergraph $H, k(H ; r)$ may be much smaller than $k\left(K_{r \chi(H)}^{(r+1)} ; r\right)$. For example, fix $r$ and let $H$ be the complete $r$-uniform hypergraph on $\ell$ vertices $K_{\ell}^{(r)}$. We have $k(H ; r)=1$ but $r \chi(H)>\ell$ so $k\left(K_{r \chi(H)}^{(r+1)} ; r\right) \geq \min \left\{k: \ell \nrightarrow(r+1)_{k}^{r}\right\} \rightarrow \infty$ as $\ell$ increases. 
Proof of Theorem 4.3. Let $C_{1}: V \rightarrow \llbracket n \rrbracket$ be the given proper colouring of $V$. Let $C_{2}:\left[\llbracket n \rrbracket \rrbracket^{r} \rightarrow\right.$ $\llbracket k \rrbracket$ be an $r$-subset colouring without monochromatic set in $[\llbracket n \rrbracket]^{r+1}$.

In order to describe the colouring, we need some more definitions. An (m-part) partition of $r$ is a non-increasing sequence of positive integers $\left(r_{0}, \ldots, r_{m-1}\right) \in(\mathbf{N} \backslash\{0\})^{m}$ with $\sum_{i \in \llbracket m \rrbracket} r_{i}=r$. When $m>1$, this partition is balanced if $r_{0}=\cdots=r_{m-1}$, and almost balanced if $r_{0}=\cdots=$ $r_{m-2}$ and $r_{m-1}=r_{0}-1$.

To every partition $R=\left(r_{0}, \ldots, r_{m-1}\right)$ we associate an ordered pair in $\llbracket 2 \rrbracket^{2}$ :

$$
C_{0}(R)=\left(\left\{\begin{array}{ll}
0 & \text { if } m+r_{m-1} \text { is even, } \\
1 & \text { otherwise }
\end{array},\left\{\begin{array}{ll}
0 & \text { if } r_{m-1}=1 \\
1 & \text { otherwise }
\end{array}\right) .\right.\right.
$$

In addition, given $C_{0}(R)=(a, b)$ we define $\overline{C_{0}}(R)=k+2 a+b \in\{k, k+1, k+2, k+3\}$.

Finally, the histogram of a sequence $\left(x_{i}\right)_{i \in[r]}$ is the unique partition $\left(r_{0}, \ldots, r_{m-1}\right)$ of $r$ such that $\left(x_{i}\right)_{i \in[r]}$ contains exactly $m$ distinct values $y_{0}, \ldots y_{m-1}$, and $y_{i}$ appears exactly $r_{i}$ times. For example, the histogram of the 6 -sequence $(9,7,7,7,15,15)$ is $(3,2,1)$, a partition of 6 .

We are ready to define the $r$-subset colouring $C:[V]^{r} \rightarrow \llbracket k+5 \rrbracket$. Given $\left\{x_{1}, \ldots, x_{r}\right\} \in[V]^{r}$, write $c_{i}=C_{1}\left(x_{i}\right)$ for every $i \in[r]$. Let $S=\left(r_{0}, \ldots, r_{m-1}\right)$ be the histogram of the sequence $\left(c_{i}\right)_{i \in[r]}$. We let

$$
C\left(\left\{x_{1}, \ldots, x_{r}\right\}\right)= \begin{cases}C_{2}\left(\left\{c_{i}: i \in \llbracket r \rrbracket\right\}\right) \in \llbracket k \rrbracket & \text { if } S=(1,1, \ldots, 1), \\ k+4 & \text { if } S \text { is almost balanced } \\ & \text { and } \min \left\{c_{i}: i \in \llbracket r \rrbracket\right\} \text { appears } \\ \bar{C}_{0}(S) \in\{k, k+1, k+2, k+3\} & \text { exactly } r_{m-1} \text { times, } \\ \text { otherwise. }\end{cases}
$$

For example, if $r=5, n>15$, and some 5 -subset $\left\{x_{1}, \ldots, x_{5}\right\}$ of $V$ gets in $C_{1}$ the colours $(2,3,3,3,15)$, then $S=(3,1,1)$, and so $C\left(\left\{x_{1}, \ldots, x_{5}\right\}\right)=k+2 \cdot 0+0=k$, since $m+r_{m-1}=3+1$ is even, and $r_{m-1}=r_{2}=1$.

Validity of the colouring. Without loss of generality it suffices to prove that any hyperedge of size $r+1$, that is $e=\left\{x_{1}, \ldots, x_{r+1}\right\} \in E$, contains two $r$-subsets receiving distinct colours in the colouring $C$. Let $R$ be the histogram of the sequence $\left(C_{1}\left(x_{i}\right)\right)_{i \in[r+1]}$.

Let us first eliminate one of the possible cases in the colouring.

Observation 4.6. The hyperedge e is not monochromatic in the colour $k+4$.

Proof of Observation 4.6. If $R$ is balanced, then clearly $e$ contains an $r$-subset that is not coloured $k+4$. If $R$ is not balanced, assume to the contrary that all $r$-subsets of $e$ are coloured $k+4$. In particular their histograms are all almost balanced.

Let $(\underbrace{a, \ldots, a}_{m+1 \text { places }}, a-1)$ be the almost balanced histogram of some $r$-subset of $e$ with $m \geq 0$ and $a \geq 2$. Then $R$ is either (I) $(a+1, \underbrace{a, \ldots, a}_{m \text { places }}, a-1)$ or (II) $(\underbrace{a, \ldots, a}_{m+1 \text { places }}, a-1,1)$.

In case (I), we have $m>0$ as otherwise depending on whether $a>2$ some $r$-subset has the histogram (3) or $(a+1, a-2)$, and neither is almost balanced. Then we must have $m=1$, $a=2$ and $R=(3,2,1)$, but in this case $r=5$ and $e$ contains a 5 -subset with histogram $(3,1,1)$, which is not almost balanced either. 
In case (II), as by assumption $(\underbrace{a, \ldots, a}_{m \text { places }} a-1, a-1,1)$ is almost balanced, we have $m=0$ and $a=3$. Hence $R=(3,2,1)$, still a contradiction.

It follows from Observation 4.6 that whenever $e$ contains an $r$-subset coloured with $k+4$, $e$ is not monochromatic in the colouring $C$. Hence, in the analysis below (specifically, in cases $3-5)$, no $r$-subset of $e$ is ever monochromatic in colour $k+4$.

As $e$ is not monochromatic under $C_{1}$ we have $m \geq 2$. Five cases cover all remaining possible values of $R=\left(r_{0}, \ldots, r_{m-1}\right)$. Let $\delta_{i j}$ be 1 if $i=j$ and 0 otherwise.

- Case 1: $r_{0}=r_{m-1}=1$, i.e. the $C_{1}\left(x_{i}\right)$ are all distinct. Then, by the definition of $C_{2}$, $\left\{C_{1}\left(x_{i}\right): i \in[r+1]\right\}$ contains two $r$-subsets that admit two distinct colours in $C_{2}$, and therefore also in $C$.

- Case 2: $r_{0}=r_{m-1}>1$. In this case, some $r$-subset of $e$ is coloured in $C$ with $k+4$, and by Observation 4.6 we are done.

- Case 3: $r_{0}>r_{m-1}>1$. In this case, some $r$-subset of $e$ has the histogram $R_{1}=$ $\left(r_{0}, \ldots, r_{m-2}, r_{m-1}-1\right)$, while another $r$-subset of $e$ has the histogram $R_{2}=\left(r_{i}-\delta_{i j}\right)_{i \in \llbracket m \rrbracket}$ for some $j<m-1$. Since $C_{0}\left(R_{1}\right)$ and $C_{0}\left(R_{2}\right)$ differ in the first coordinate, $\bar{C}_{0}\left(R_{1}\right) \neq$ $\bar{C}_{0}\left(R_{2}\right)$ hence these two $r$-subsets admit two distinct colours in $C$.

- Case 4: $r_{m-2}>r_{m-1}=1$. In this case, some $r$-subset of $e$ has the histogram $R_{1}=$ $\left(r_{0}, \ldots, r_{m-2}\right)$, while another $r$-subset of $e$ has the histogram $R_{2}=\left(r_{0}, r_{1}, \ldots, r_{m-2}-1,1\right)$. Since $C_{0}\left(R_{1}\right)$ and $C_{0}\left(R_{2}\right)$ differ in the second coordinate, $\bar{C}_{0}\left(R_{1}\right) \neq \bar{C}_{0}\left(R_{2}\right)$ hence these two $r$-subsets admit two distinct colours in $C$.

- Case 5: $r_{0}>r_{m-2}=r_{m-1}=1$. In this case, some $r$-subset of $e$ has the histogram $R_{1}=\left(r_{0}, \ldots, r_{m-2}\right)$, while another $r$-subset of $e$ has the histogram $R_{2}=\left(r_{i}-\delta_{i j}\right)_{i \in \llbracket m \rrbracket}$ for some $j<m-2$. Since $C_{0}\left(R_{1}\right)$ and $C_{0}\left(R_{2}\right)$ differ in the first coordinate (note that $\left.r_{m-1}=r_{m-2}=1\right), \bar{C}_{0}\left(R_{1}\right) \neq \bar{C}_{0}\left(R_{2}\right)$ hence these two $r$-subsets admit two distinct colours in $C$.

Finally, note that for specific values of $r$, some colours are left unused: For $r=2$, the colours used are in $\llbracket k \rrbracket \cup\{k+2\}$. For $r=3$, they are in $\llbracket k \rrbracket \cup\{k+1, k+2, k+4\}$. Finally, if $r+1$ is prime then $R$ cannot be balanced without being $(1,1, \ldots, 1)$. In this case we can define $C$ without using the colour $k+4$ (whose role in the proof is to handle balanced partitions of $r+1$ ). This justifies having the number of colours $f(r)$ as in the statement of the theorem.

For the next result, let $\log x=\max \{1, \log x\}$.

Corollary 4.7. For $n \geq r \geq 2$, we have

$$
\frac{1}{3} \cdot \frac{\log ^{(r-1)}(r n)}{\log ^{(r)}(r n)}+o(1) \leq k(n, r) \leq \frac{2}{\alpha} \log ^{(r-1)}(r n)+\frac{5 r}{2}+O(1) .
$$

Proof. Combining Observation 4.1 and Theorem 4.2, we have

$$
\min \left\{k: r_{k}(r+1 ; r)>r n\right\} \leq k(n, r) \leq \min \left\{k: r_{k}(r+1 ; r)>r n\right\}+5 .
$$


have

For $r n$ large enough, let $k_{0}=\left\lceil\frac{2}{\alpha}\left(\log ^{(r-1)}(r n)-\beta\right)+\frac{5 r}{2}\right\rceil \geq\lceil 5 r / 2\rceil$. By Corollary 1.3 , we

$$
r_{k_{0}}(r+1 ; r)>\operatorname{twr}_{r}\left(\frac{\alpha}{2} \cdot\left(k_{0}-\frac{5 r}{2}\right)+\beta\right) \geq r n
$$

thus $k(n, r) \leq k_{0}+5$.

Similarly, also for large $r n$, let $k_{1}=\left\lfloor\frac{1}{3} \cdot \frac{\log ^{(r-1)}(r n)}{\log ^{(r)}(r n)}\right\rfloor$. By Theorem 3.2 we have $r_{k_{1}}(r+1 ; r)<$ $\operatorname{twr}_{r}\left(3 k_{1} \log k_{1}\right) \leq r n$. By the monotony of $r_{k}(r+1 ; r)$ in $k$ (Observation 3.1), we conclude $k(n, r)>k_{1}$.

\section{References}

[1] M. Axenovich, A. Gyárfás, H. Liu, and D. Mubayi. "Multicolor Ramsey numbers for triple systems". In: Discrete Mathematics 322 (2014), pp. 69-77. ISSN: 0012-365X. DOI: $10.1016 / j$.disc.2014.01.004.

[2] M. Bruce, M. Budden, and J. Hiller. "Lexicographic products of $r$-uniform hypergraphs and some applications to hypergraph Ramsey theory". In: Australasian journal of combinatorics 70.3 (2018), pp. 390-401.

[3] F. R. K. Chung and C. M. Grinstead. "A survey of bounds for classical Ramsey numbers". In: Journal of Graph Theory 7.1 (1983), pp. 25-37. DOI: 10.1002/jgt.3190070105.

[4] D. Conlon, J. Fox, and B. Sudakov. "An improved bound for the stepping-up lemma". In: Discrete Applied Mathematics 161.9 (2013). Jubilee Conference on Discrete Mathematics, pp. 1191-1196. ISSN: 0166-218X. DOI: 10.1016/j.dam.2010.10.013.

[5] D. Duffus, H. Lefmann, and V. Rödl. "Shift graphs and lower bounds on Ramsey numbers $r_{k}(l ; r)$ ". In: Discrete Mathematics 137.1 (1995), pp. 177-187. ISSN: 0012-365X. DOI: 10 . 1016/0012-365X (93)E0139-U.

[6] P. Erdôs, A. Hajnal, and R. Rado. "Partition relations for cardinal numbers". In: Acta Mathematica Academiae Scientiarum Hungaricae 16 (1965), pp. 93-196. DOI: 10.1007/ BF01886396.

[7] P. Erdôs and R. Rado. "Combinatorial theorems on classifications of subsets of a given set". In: Proceedings of the London Mathematical Society 2.3 (1952), pp. 417-439.

[8] P. Erdôs and A. H. Stone. "On the structure of linear graphs". In: Bulletin of the American Mathematical Society 52.12 (1946), pp. 1087-1091. DOI: bams/1183510246.

[9] P. Erdős, A. Hajnal, A. Máté, and R. Rado. Combinatorial set theory: partition relations for cardinals. Studies in logic and the foundations of mathematics 106. North-Holland and Akadémiai Kiadó, 1984. ISBN: 0-444-86157-2.

[10] H. Fredricksen and M. M. Sweet. "Symmetric Sum-Free Partitions and Lower Bounds for Schur Numbers". In: The Electronic Journal of Combinatorics 7, R32 (2000). DOI: $10.37236 / 1510$.

[11] R. L. Graham, B. L. Rothschild, and J. H. Spencer. Ramsey Theory. 2nd ed. WileyInterscience Series in Discrete Mathematics. John Wiley \& Sons, Inc., 1990. ISBN: 0-47105997-8. 
[12] B. D. McKay and S. P. Radziszowski. "The First Classical Ramsey Number for Hypergraphs is Computed". In: Proceedings of the Second Annual ACM-SIAM Symposium on Discrete Algorithms. SODA '91. San Francisco, California, USA: Society for Industrial and Applied Mathematics, 1991, pp. 304-308. ISBN: 0897913760. DOI: 10.5555/127787. 127844 .

[13] S. Radziszowski. "Small Ramsey Numbers". Version 16. In: The Electronic Journal of Combinatorics, DS1 (Dynamic Surveys 2021). DOI: 10.37236/21.

[14] F. P. Ramsey. "On a Problem of Formal Logic". In: Proceedings of the London Mathematical Society s2-30.1 (Jan. 1930), pp. 264-286. ISSN: 0024-6115. DOI: 10.1112/plms/s2-30.1. 264 .

[15] K. S. Sarkaria. "Heawood inequalities". In: Journal of Combinatorial Theory, Series A 46 (1987), pp. 50-78.

[16] K. S. Sarkaria. "On coloring manifolds". In: Illinois Journal of Mathematics 25.3 (1981), pp. 464-469.

[17] K. S. Sarkaria. "On neighbourly triangulations". In: Transactions of the American Mathematical Society 277.1 (1983), pp. 213-239.

\section{A Infinitary Ramsey combinatorics}

In this section we sketch the modifications needed to extend our stepping-up lemma to infinite cardinals.

We work in Zermelo-Fraenkel set theory with choice (ZFC), and we use von Neumann ordinals (so for integers we have $n=\llbracket n \rrbracket$, more generally any ordinal $\alpha=\{\beta: \beta<\alpha\}$, and the cardinal of any set $S$ is $|S|=\min \{\alpha$ ordinal: $\alpha \simeq S\}$ ). As usual we also let $\omega=|\mathbf{N}|=\mathbf{N}$. We write + and $\dot{+}$ for cardinal and ordinal addition respectively.

Let $r \geq 2$ be an integer, let $\tau>0$ be an ordinal, $\kappa>0$ and $\lambda_{\xi} \geq r+1$ (for every $\xi<\tau)$ be cardinals. Extend the relation $\nrightarrow$ previously defined for integers by calling $X \subset \kappa$ is monochromatic in colour $\xi<\tau$ under $f:[\kappa]^{r} \rightarrow \tau$ if $f "[X]^{r} \subset\{\xi\}$ and writing

$$
\kappa \nrightarrow\left(\lambda_{\xi}\right)_{\xi<\tau}^{r}
$$

if for some $f:[\kappa]^{r} \rightarrow \tau$ there does not exist $\xi<\tau$ and a subset $X \subset \kappa$ monochromatic in $\xi$ such that $|X|=\lambda_{\xi}$.

Assuming the above relation holds and $r \geq 3$, Erdôs et al. [9, chap. 24] prove

$$
2^{\kappa} \nrightarrow\left(\left(\lambda_{\xi}+1\right)_{\xi<\tau},(r+2)_{2^{r}+2^{r-1}-4}\right)^{r+1},
$$

which is shorthand for $2^{\kappa} \nrightarrow\left(\lambda_{\xi}^{\prime}+1\right)_{\xi<\tau \dot{+}\left(2^{r}+2^{r-1}-4\right)}^{r+1}$ where $\lambda_{\xi}^{\prime}$ is $\lambda_{\xi}$ for $\xi<\tau$ and otherwise $r+1$. We can strengthen this result somewhat:

Theorem A.1. Let $r \geq 3$ be an integer, let $\kappa>0$ be a cardinal, $\tau>0$ an ordinal and $\lambda_{\xi} \geq r+1$ $(\xi<\tau)$ cardinals.

$$
\text { If } \kappa \nrightarrow\left(\lambda_{\xi}\right)_{\xi<\tau}^{r} \text { then } 2^{\kappa} \nrightarrow(\left(\lambda_{\xi}+1\right)_{\xi<\tau}, \underbrace{r+2, \ldots, r+2}_{\begin{array}{c}
\eta(r) \text { terms, } \\
\text { each } r+2
\end{array}})^{r+1} \text {, }
$$

with the integer $\eta(r) \leq 3$ defined above in (1). 
To the best of our knowledge, this improves upon known results in two cases: when $\kappa$ and all $\lambda_{\xi}$ are finite (corresponding to our previous finite results), and when $r=3, \kappa$ is infinite, $\lambda_{0}$ is infinite and singular and all other $\lambda_{\xi}$ are finite. In all other cases, the book by Erdős et al. contains stronger statements.

Proof sketch. The hypothesis means that there exists $f_{r}:[\kappa]^{r} \rightarrow \tau$ for which none of the colour classes has a monochromatic set of cardinality $\lambda_{\xi}$. Due to the bijection $2^{\kappa} \simeq|\mathcal{P}(\kappa)|$ it suffices to exhibit $f_{r+1}:[\mathcal{P}(\kappa)]^{r+1} \rightarrow \tau \dot{+} \eta(r)$ for which none of the first $\tau$ colour classes has a monochromatic set of cardinality $\lambda_{\xi}+1$, and none of the subsequent ones has a monochromatic set of cardinality $r+2$.

To do so define the first discrepancy between two sets of ordinals $x$ and $y$ with $x \neq y$ as

$$
s(x, y)=\min ((x \cup y) \backslash(x \cap y)),
$$

which is the well-defined minimum of a non-empty set of ordinals. From there if $\sigma \subset \mathcal{P}(\kappa)$ is a set of subsets of $\kappa$ and $|\sigma| \geq 2$ we define its first splitting index as $s(\sigma)=\min \left(s "[\sigma]^{2}\right)$, a well-defined ordinal in $\kappa$.

This gives essentially the same partition as before with $\sigma=\left(\sigma_{0} \mid \sigma_{1}\right)$ where $\sigma_{0}=\{S \in$ $\sigma: s(\sigma) \notin S\}$ and $\sigma_{1}=\sigma \backslash \sigma_{0}$. It admittedly differs from the previous finitary construction in a minor way, as we now compare least significant bits, but when restricted to finite sets it is functionally equivalent. This small modification is necessary; using maxima in the definitions above would make these quantities ill-defined.

A caterpillar is a set of any size without a four-subset of the form $(L \mid R)$ with $|L|=|R|=2$. If $\sigma=\left(\sigma_{0} \mid \sigma_{1}\right)$ is a caterpillar then at least one of $\sigma_{0}$ and $\sigma_{1}$ is a singleton.

For any caterpillar $T \subset \mathcal{P}(\kappa)$ let $\delta(T)=\left\{s(x, y):\{x, y\} \in[T]^{2}\right\}=s "[T]^{2}$.

We (still) have $[\delta(T)]^{r}=\delta "[T]^{r+1}$, extending Observation 2.4. This infinite case is not harder to prove since

$$
[\delta(T)]^{r}=\bigcup_{\substack{Y \subset T \\|Y|<\omega}}[\delta(Y)]^{r}=\bigcup_{\substack{Y \subset T \\|Y|<\omega}} \delta "[Y]^{r+1}=\delta "\left(\bigcup_{\substack{Y \subset T \\|Y|<\omega}}[Y]^{r+1}\right)=\delta "[T]^{r+1} .
$$

Similarly, the equality $|T|=|\delta(T)|+1$ still holds for every caterpillar $T$ by transfinite induction.

We re-use the same colouring scheme. Let $S \in[\mathcal{P}(\kappa)]^{r+1}$. If $S \in \mathcal{C}$ then $\delta(S) \in[\kappa]^{r}$, and we let $f_{r+1}(S)=f_{r}(\delta(S))<\tau$. If $S \notin \mathcal{C}$, then $S$, being finite, has a type, so we simply colour it as before.

As before, let us check the validity of this colouring only for odd $r$.

Let $X \subset \mathcal{P}(\kappa)$ be monochromatic in colour $\xi$ under $f_{r+1}$. We will show that $|X| \leq \lambda_{\xi}$ (if $\xi<\tau$ ) or $|X| \leq r+1$ (for $\xi \in\{\tau, \tau+1\}$ ). As $r+1 \leq \lambda_{\xi}$ we may already assume $|X|>r+1$.

First consider the case when $X \in \mathcal{C}$. Then $[X]^{r+1} \subset \mathcal{C}$, and thus $\{\xi\}=f_{r+1} "[X]^{r+1}=$ $f_{r} "\left(\delta "[X]^{r+1}\right)<\tau$, so we have to show $|X| \leq \lambda_{\xi}$.

The set $\delta(X) \subset \kappa$ is monochromatic in colour $\xi$ under $f_{r}$, as

$$
f_{r} "[\delta(X)]^{r}=f_{r} "\left(\delta "[X]^{r+1}\right)=\{\xi\} .
$$

This fact implies that $|X|=|\delta(X)|+1<\lambda_{\xi}+1$.

The other case to consider is when $X \notin \mathcal{C}$. We show that then $|X| \leq r+1$ regardless of $i$. (Recall that $r+1 \leq s_{i}$ always.) Suppose to the contrary that $|X| \geq r+2$, and we can assume $|X|=r+2$. Now everything is finite, and works as before. 


\section{B Lower bounds on $r_{k}(3 ; 2)$}

Here is the justification for the lower bounds on $r_{k}(3 ; 2)$ used in the proof of Corollary 1.3 .

Theorem B.1. There is an absolute constant $C>0$ such that for all $k \geq 2$ we have

$$
\left\lceil C \cdot 1073^{k / 6}\right\rceil \nrightarrow(3)_{k}^{2} .
$$

Proof. A set of integers $S$ is sum-free if $(S+S) \cap S$ is empty. The Schur number $s_{k}$ is the largest number for which $\left\{1,2, \ldots, s_{k}\right\}$ has a partition into $k$ sum-free subsets. By Schur's Ramsey theorem, $s_{k} \nrightarrow(3)_{k}^{2}$ (see e.g. 11, chap. 4.5]). For any fixed positive integer $l$ and $k \geq l$ we have [3]

$$
s_{k} \geq C_{l} \cdot\left(2 s_{l}+1\right)^{k / l} .
$$

Finally, $s_{6} \geq 536[10]$. 